\title{
Adverse drug reaction
}

\section{Recurrent aseptic meningitis following non- steroidal anti-inflammatory drugs - a reminder}

\author{
R A Seaton, A J France
}

\begin{abstract}
Summary
Non-steroidal anti-inflammatory drugs (NSAIDs) are rarely associated with sideeffects affecting the central nervous system. A case of NSAID-induced recurrent aseptic meningitis is presented. Seven episodes of aseptic meningitis were documented in the patient's life-time (up to the age of 30). General practitioners' records available for the latest four episodes showed that a NSAID (naproxen, piroxicam or diclofenac) was prescribed in the month prior to admission on each occasion. The patient was symptom free for a 3-year period during which no NSAID was prescribed. Clinicians should always elicit a careful drug history (including over-the-counter medications) in patients with aseptic meningitis and be aware of this unusual side-effect of NSAIDs.
\end{abstract}

Keywords: non-steroidal anti-inflammatory drugs; meningitis; adverse drug reaction

Aseptic meningitis is a rare condition associated with collagen vascular and autoimmune disease and a number of drugs and chemicals. Drugs that have been implicated include the antimicrobial agents trimethoprim, sulfamethoxazole, ciprofloxacin, penicillin and isoniazid, the monoclonal antibody muromonab-CD3, azathioprine, carbamazepine, cytosine arabinoside, immune globulin and phenazopyridine. ${ }^{1}$ The association between non-steroidal antiinflammatory drugs (NSAIDs) and aseptic meningitis was first observed in 1978 in a patient with systemic lupus erythematosus (SLE) receiving ibuprofen. ${ }^{2}$ Thereafter episodes of aseptic meningitis have been observed in patients with or without collagen vascular or autoimmune disease following a number of different NSAIDs including suldinac, tolmetin and naproxen but most commonly ibuprofen. ${ }^{3} \mathrm{Al}-$ though this association is now well described, cases of aseptic meningitis are sufficiently rare and NSAID use so common that the association may be overlooked. We present a patient with seven documented episodes of aseptic meningitis in whom the association with NSAIDs was not recognised until his final admission.

\section{Case report}

A 30-year-old man presented with 3 days of headache, nausea, neck pain and photophobia.
Two days prior to the onset of symptoms he had taken diclofenac retard for back pain. Examination revealed nuchal rigidity and photophobia, cerebrospinal fluid (CSF) was opaque with an opening pressure of $>300 \mathrm{~mm}$, protein concentration was $1.62 \mathrm{~g} / 1$ and CSFto-blood glucose ratio was 0.51 . Microscopy demonstrated 665 mononuclear cells $/ \mathrm{mm}^{3}$ but bacterial and viral culture were negative.

He had six previous documented episodes of aseptic meningitis. Admissions were stereotyped with symptoms improving rapidly following lumbar puncture. Serological tests for herpes viruses, rheumatoid factor and antinuclear antibody were negative. General practitioner records were available for the previous 6 years. All four episodes of aseptic meningitis within this 6-year period had occurred within the month following the prescription of a NSAID (naproxen, piroxicam and diclofenac) for mechanical back pain, suprascapular tendonitis and tennis elbow. In the final three admissions diclofenac was prescribed 6,5 and 2 days prior to the onset of headache. Over a 3-year period where NSAIDs were not prescribed the patient did not present to his general practitioner with headaches. Following the final admission the patient was advised to avoid all NSAIDs in the future. Re-challenge with a NSAID in hospital was not undertaken following discussion with the patient. In the 2 years since there have been no further episodes of aseptic meningitis.

\section{Discussion}

In any patient presenting with fever, headache and meningism with CSF findings consistent with aseptic meningitis, an infective cause should always be sought and treated empirically with antimicrobials. In the rare patient in whom recurrent bouts of aseptic meningitis occur, a chemical trigger should be suspected and a careful drug history, including over-thecounter medications, should be taken. In those in whom a trigger cannot be identified and a predisposing condition, such as autoimmune disease, is not suspected the diagnosis 'Mollaret's meningitis' is usually made. The aetiology of this condition is uncertain, although some cases have been associated with recurrent herpes simplex infections (types 1 and 2) demonstrable by amplification of viral DNA in the $\mathrm{CSF}^{4}{ }^{5}$

In the patient presented there was a temporal association between the prescription of either diclofenac, piroxicam or naproxen and 
the development of aseptic meningitis. Most cases of NSAID-induced aseptic meningitis appear to be agent-specific as rechallenge with other agents does not tend to result in aseptic meningitis. ${ }^{3}$ At least one other patient, however, has had aseptic meningitis related to more than one agent (suldinac and ibuprofen). ${ }^{6}$ The mechanism for this adverse reaction is unclear. The association is most commonly seen in women with SLE although male patients without underlying pathology have also been described. The most plausible explanation for the association is a hypersensitivity reaction involving the meninges. Evidence for this is mainly circumstantial: the symptoms develop rapidly following the ingestion of the drug, incubation periods become shorter with repeat attacks and recovery is rapid on stopping the drug. A minority of patients also may describe flushing and other symptoms of a hypersensitivity type reaction. ${ }^{13}$ Prostaglandin inhibition as a cause is unlikely in most cases of NSAIDinduced aseptic meningitis since rechallenge with an alternate NSAID tends not to repro-

1 Chaudhry HJ, Cunha BA. Drug-induced aseptic meningitis. Postgrad Med 1991;90:65-70.

2 Widener HI, Littman BH. Ibuprofen-induced meningitis in systemic lupus erythematosus. FAMA 1978;239:1062-4.

3 Hoppmann RA, Peden JG, Ober SK. Central nervous Hoppmann RA, Peden JG, Ober SK. Central nervous system side effects of non-steroidal anti-
drugs. Arch Intern Med 1991;151:1309-13.

4 Yamamoto LJ, Tedder DG, Ashley R, Levin MJ. Herpes simplex virus type 1 DNA in cerebrospinal fluid of a patien with Mollaret's meningitis. N Engl f Med 1991;325:1082-5.

\section{Summary points}

- aseptic meningitis is a rare condition associated with a number of drugs and autoimmune diseases

- NSAIDs are an important cause of aseptic meningitis and recurrent bouts may result from repeat prescribing

- a careful drug history should be taken in all patients presenting with meningitis when an organism is not isolated

duce the symptoms although this cause cannot be discounted in our patient. Other central nervous system effects of NSAIDs are well described, particularly in the elderly and these include acute psychosis and cognitive deficits. ${ }^{3}$ Again the pathophysiology of these adverse effects are not known.

Since NSAIDs are commonly dispensed 'over-the-counter', their role in the aetiology of aseptic meningitis may be underestimated.

5 Cohen BA, Rowley AH, Long CM. Herpes simplex type 2 in a patient with Mollaret's meningitis: demonstration by polymerase chain reaction. Ann Neurol 1994;35:112-6.

6 Ruppert GB, Barth WF. Tolmetin-induced aseptic meningitis. $\mathcal{F} A M A 1981 ; 245: 67-8$

\section{Images in medicine}

\section{Discolouration of permanent teeth and enamel hypoplasia due to tetracycline}

An 18-year-old girl reported to dental outpatients with complaints of persistent yellowish-brown discolouration and deformity of teeth (figure) of many years duration. She had been given repeated courses of tetracycline hydrochloride for respiratory tract infections from 2-7 years of age. There was yellow-brown discolouration and enamel hypoplasia of the incisors, canines, and premolars. When given during the second half of pregnancy, in infancy, and in childhood up to eight years of age, tetracyclines cause permanent yellow-brown discolouration and enamel hypoplasia of permanent teeth. This results from administration of tetracyclines during the period of crown development, as they chelate with calcium and are deposited in the dentin. Deposition in enamel is minimal. The extent of discolouration depends on how close to the dentino-enamel junction the deposits occur. Enamel is thinnest

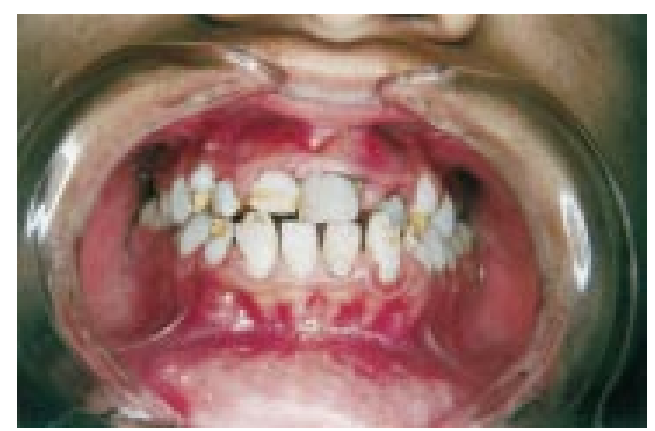

at the cervical part of the tooth, therefore discolouration is first seen in this area.

A S KASHYAP

H S SHARMA

Department of Medicine, Armed Forces Medical College, Pune 411040, India

Accepted 5 July 1999 